\title{
Characterization of Micronized Wood and Energy-size Relationship in Wood
}

\section{Comminution}

\author{
Jinxue Jiang ${ }^{\mathrm{a},}{ }^{*}$, Jinwu Wang $^{\mathrm{b}}$, Xiao Zhang ${ }^{\mathrm{c}}$, Michael Wolcott ${ }^{\mathrm{a}}$ \\ ${ }^{a}$ Composite Materials and Engineering Center, Washington State University, Pullman, WA, 99164, USA \\ ${ }^{\mathrm{b}}$ Forest Products Laboratory, United States Department of Agriculture Forest Service, Orono, ME, 04469, USA. \\ ${ }^{\mathrm{c}}$ Voiland School of Chemical Engineering and Bioengineering, Washington State University, Richland, WA, 99354, \\ USA \\ *Corresponding author. Tel.: +1 (509)-715-9838. E-mail: jinxue.jiang@wsu.edu (J. Jiang).
}

\begin{abstract}
Mechanical milling demonstrates potential in the pretreatment arena to valorize lignocellulosic biomass because it eliminates chemicals and simplifies processing. The development of physical properties and energy consumption for generating micronized particles are key factors affecting potential use. This study investigated the effect of input moisture content, and feedstock particle size on developing physical characteristics of micronized particles and the resultant specific energy consumption in the milling process. Wood particles with different sizes were effectively comminuted to less than $20-\mu \mathrm{m}$ within several minutes using a vibratory ring and puck mill. Moisture content was found to be a key factor influencing the development of particle morphology and crystallinity. Lower moisture content resulted in much rounder particles with lower crystallinity, while higher moisture content resulted in the micronized particles with larger aspect ratio and crystallinity. Crystallinity index and median aspect ratio of the micronized particles were linearly correlated. The particle size change during milling was highly correlated to the specific energy consumption of milling through the Rittinger's model $\left(0.91<\mathrm{R}^{2}<0.96\right)$. Input moisture content and feed size were found to affect the energy intensity of grinding woody biomass. The Rittinger's constant was a good indicator of the material performance in this area. The results will provide a guidance for preferred milling conditions as well as designing scalable micronizing mills.
\end{abstract}


Key words: fine milling, woody biomass, morphology, crystallinity, specific energy consumption, particle size distribution, aspect ratio.

\section{Introduction}

The development of sustainable biofuels from woody biomass is becoming increasingly important for diversifying our transportation energy portfolio and mitigating the global greenhouse gas emission [1]. Both biochemical and thermochemical biorefinery processes have encountered challenges for commercial implementation, including the robust biomass recalcitrance and the need for an integrated feedstock supply. Depot-based technologies may be useful for transforming bulky biomass into an interchangeable feedstock for multiple applications; e.g. pretreated wood, or fermentable sugars [2]. Such an approach can potentially reduce risk and logistics costs of feedstock supply for centralized downstream processes. An appropriate pretreatment plays a key role for such depots by facilitating the potential distributed production of cellulosic sugars.

Mechanical deconstruction of biomass offers potential for streamlining processes and enabling supply chains that utilize existing forest products facilities, provide flexibility to operate at various scales, eliminate chemical input, and reduce or eliminating waste water treatment requirements [3,4]. Mechanical milling is a common method of altering physical properties of substrates (e.g., particle size, aspect ratio and cellulose crystallinity, etc.) and can significantly facilitate either biochemical or thermochemical conversions. Reduced particle size is beneficial to biomass conversion by improving particle reactivity and heat / mass transfer efficiency $[3,5]$. The aspect ratio of particles is a crucial factor for influencing biomass pyrolysis efficiency, though the relationship between particle shape and biochemical conversion efficiency remains 
elusive [6-8]. However, it is known that the cellulose crystallinity in lignocellulosic biomass influences the rate and extent of enzymatic digestion [3,9]. The physical properties of milled biomass can be controlled by milling conditions; e.g. the moisture content of feed material, and feedstock particle size. Kobayashi et al. [10] reported that wood powder pulverized with a vibratory mill at $3 \%$ moisture content had a reduced crystallinity of $14.3 \%$, while wood powder ground from $11 \%$ moisture content resulted in a crystallinity of $37.7 \%$. Even though the exact fracture mechanism of particles in the milling chamber is unknown, Gil et al. [11] have shown that the feedstock particle size influenced the particle morphology of final products in hammer milling. According to Gil et al, single particle breakage in an impact milling is related to the particle volume and contact area upon impact; both of which are determined by the particle size. Thus, a larger particle size increases the probability of structure fragmentation, leading to rapid particle size reduction. Despite a significant amount of information obtained on the effect of milling conditions on the physical properties of coarsely milled particles from several types of mills $[4,12]$, a systematic understanding of the effect of milling conditions on producing fine particle fractions (i.e., partial size below $100 \mu \mathrm{m}$ ) and their ensuing physical properties is lacking. This finely milled biomass with an average particle size below $100 \mu \mathrm{m}$ is termed here as micronized biomass. These micronized particles exhibiting a significant level of cell wall disintegration, have shown to be susceptible to enzymatic hydrolysis [13-15]. Such micronized biomass was also found to be easier thermochemical conversion (e.g. pyrolysis) with lower degradation temperature and lower thermal degradation activation energies, facilitating production of high-value furfurals, mainly due to their high reactivity[16]. Additionally, micronized wood is highly preferable for industrial application of low-cost natural fiber-based thermoplastic composites, as it provides high surface area for better reinforcement[17]. 
Conventional milling technology like hammer milling is incapable of providing sufficient size reduction to produce a micronized particle [18]. In contrast, micronized wood is commonly prepared using vibratory milling $[19,20]$.

It is apparent that a considerable amount of energy is consumed during size reduction of lignocellulosic biomass [21,22]. A number of previous studies have attempted to establish the relationship between energy consumption and particle size reduction during biomass milling. For example, a power-law relation was proposed to correlate energy consumption with product particle size (e.g., screen size) or the biomass comminution ratios $[23,24]$. Inspired by the milling theories proposed to delineate the specific relationship for milling ores, Temmerman et al. characterized the energy-size relationship with the Rittinger's model for hammer milling wood chip and pellet. Among these studies, the Rittinger's model was recognized as the best approach to describe fine milling performance, as well as a good indicator for assessing a given comminution system [25-27]. However, predicting the energy consumption for micronized wood by mechanical milling with different operational conditions is still lacking. Therefore, modeling the energy consumption of fine wood milling with the size characteristics will be the first step to optimize the milling process for potential industrial application.

The main goal of this study is to delineate the physical property development of micronized particles under various milling conditions and correlate size reduction with energy consumption of the process. The experiments focus on exploring effects of moisture content and feed size for the starting material on changes in particle morphology, cellulose crystallinity, and specific energy consumption of the fine milling process. The knowledge gained in this study will aid in developing potential approaches and strategies for producing a micronized biomass substrate in an economical manner with mechanical pretreatment. 


\section{Materials and methods}

\subsection{Material}

Clean, Douglas-fir (Pseudotsuga menziesii) wood chips were obtained locally (Vaagen Brothers Lumber Inc., Colville, WA). The as-received chips were passed through a vibrating screen with a 25.4-mm aperture and retained on a 4.75-mm screen, producing a chip with a geometric mean diameter of 10.36-mm. The screened wood chips were processed into particles by a hammer mill fitted with a $11.11-\mathrm{mm}, 6.35-\mathrm{mm}$, and $3.18-\mathrm{mm}$, screen, respectively. These screened feedstock samples were labeled as Pxx (pass) with xx being the screen size (i.e., P25, P11, P6, and P3, respectively). The processed feedstock was subsequently stored in a temperature-and humidity-conditioned room with an equilibrium moisture content (EMC) of $15 \%$ and subsequently conditioned to different target EMCs values. After final conditioning, all material was stored in sealed plastic bags and moisture content was validated using gravimetric methods according to standard protocol [28].

\subsection{Mechanical milling process}

Fine milling experiments were carried out with a high-energy vibratory ring and puck mill (Standard Ring milling, motor power 1.1-kw, Rocklab Pty Ltd, New Zealand). The milling chamber had an inner diameter of $128-\mathrm{mm}$ and height of $43-\mathrm{mm}$. The grinding media were a ring (inner diameter 78-mm, outside diameter, 100-mm, height 41-mm) and a puck (diameter 52-mm and height 41-mm). Both milling chamber and grinding media were made of tungsten carbide.

The milling experiments were designed to investigate different milling variables; these include milling time, initial moisture content, and size of feedstock. The resulting micronized wood powder was then characterized for particle size distribution, aspect ratio, and crystallinity. Milling times were varied from 2 minutes to 12 minutes with 2-minutes interval. Four initial 
moisture contents $(5,10,15$, and $30 \%$ oven dried base) were tested, using P3 particles with charge quantities of 10-grams per grinding batch. For investigating effect of the feed size on milling performance, four different initial size levels (P25, P11, P6 and P3) were tested, using conditioned moisture content of $10 \%$ samples with charge quantities of 10 -grams per milling batch.

\subsection{Measurement of specific energy consumption}

The milling energy consumption of the ring and puck mill was measured with a Fluke 1735 power logger (Fluke, USA). The active power, active energy, power factor, frequency, and time were acquired by computer. The specific grinding energy consumption was calculated using the following relation:

$E_{p}=\int_{0}^{t}\left(P_{t}-P_{0}\right) d_{t} / m=\int_{0}^{t} \Delta P_{t} d_{t} / m$

where: $E_{p}$ is the specific net energy consumption $(\mathrm{kJ} / \mathrm{kg}) ; P_{t}$ is the power consumed at time $\mathrm{t}$; $P_{0}$ is the average power consumption under idle condition measured from an empty mill; and $\mathrm{m}$ is the mass charge in $\mathrm{kg}$ of wood to be pulverized.

\subsection{Characterization of wood powder}

\subsubsection{Preparation of wood powder for analyses}

The milled wood samples were prepared for particle size distribution and aspect ratio analyses as follows. Each sample was suspended in distilled water to achieve a $0.5 \%$ solids mass concentration. For the aspect ratio measurement, a diluted powder suspension was dispersed by adding $1-\mathrm{mL}$ of a dispersant solution $(0.1 \% \mathrm{w} / \mathrm{w}$ sodium dodecyl sulfonate $)$ per $100-\mathrm{mg}$ wood [29]. The suspended samples were stirred for 1 hour with a magnetic stir rod, followed by sonication with $20 \%$ amplitude (Branson, Switzerland) for 2 minutes to ensure complete 
disintegration of particle agglomeration. The same procedure was following for measuring particle size distribution, except dispersant was not added.

\subsubsection{Particle size distribution}

The volumetric particle size distribution of the wood powders was analyzed using a laser scattering particle size analyzer (Mastersizer 3000 with Hydro LV wet sample dispersion, Malvern instrument, UK). The median size was used to represent the particles size for analysis. The dimensionless parameter $\Delta_{d}$ is used to represent the breadth of the particle size distribution, where:

$\Delta_{d}=\frac{d_{90}-d_{10}}{10 \mu m}$

and $d_{90}$ and $d_{10}$ are particle sizes corresponding to the $90 \%$ and $10 \%$ percentiles as assessed from the cumulative size distribution, respectively.

\subsubsection{Aspect ratio analysis}

The solid suspension of samples prepared as described above, was distributed on a silica wafer mounted on a metal stab. This process involved using a pipette to form a droplet with a volume of ca. $200-\mu \mathrm{L}$. After allowing the water to evaporate at ambient conditions, samples were sputtered with gold to prepare examination in a Scanning Electron Microscopy (SEM). The measurement of aspect ratio was obtained from the analysis of SEM images using ImageJ (National Institutes of Health, USA) following the procedures described elsewhere [30,31]. For each sample, about 20 SEM images containing around 30000 particles were analyzed. The aspect ratio of each particle was calculated as the ratio of the major and minor axis of the fit ellipse for the selected particle. The minimum aspect ratio value of 1 was restricted and describes a perfect and filled circle. The number based cumulative aspect ratio distribution was used to obtain the median aspect ratio $A R_{50}$, which was the value of $50 \%$ cumulative aspect ratio distribution. The 
width of aspect ratio distribution $\Delta_{A R}$ was defined as the difference between the aspect ratios corresponding to $90 \%$ and $10 \%$ values in the cumulative distribution.

\subsection{Cellulose crystallinity}

X-ray diffractograms of the milled wood particles were obtained using a powder $\mathrm{x}$-ray diffractometer (Rigaku, Miniflex 600, Japan) with a $\mathrm{Cu} \mathrm{K} \alpha(\lambda=0.154 \mathrm{~nm})$ radiation source generated at $40-\mathrm{kV}$ and $15-\mathrm{mA}$. The instrument scanning range $2 \theta$ was from 10 to $40^{\circ}$. The relative degree of cellulose crystallinity, in terms of crystallinity index (CrI), was estimated using equation as described by Segal [32]:

$\operatorname{CrI}(\%)=\left[\left(I_{002}-I_{a m}\right) / I_{002}\right] \times 100$

where $I_{002}$ is the intensity of main peak, and is the intensity due to amorphous portion evaluated as the minimum intensity between the main and secondary peaks.

\subsection{Scanning electron microscopy (SEM)}

The morphology of prepared samples as described above was analyzed by scanning electron microscopy. Images of samples were acquired typically at $20-\mathrm{kV}$ accelerating voltage using FEI Quanta 200F, field emission gun with high vacuum ETD detectors (FEI Company, Hillsboro, Oregon, USA). Prior to imaging, the suspended wet sample was air dried and sputtered with gold for good conductivity.

\section{Results and discussion}

\subsection{Effect of milling conditions on characteristics of particle morphology}

Mechanically fragmenting the lignocellulosic biomass into the micronized size range has been recognized as an essential step to enhance the substrate digestibility of mechanically pretreated substrates by others [13,33]. In this study, effectively micronized softwood was achieved using ring and puck mill under various milling conditions. The change in median 
particle size $d_{50}$ as a function of milling time for feedstock with various initial moisture contents is presented in Fig. 1A. At the early stage of milling (e.g., at the 2-minutes point), an elevated moisture content facilitates more rapid particle size reduction (Fig.1A), which is likely a result of the higher specific energy input for milling higher moisture content samples (e.g. MC30\%). A previous study reported that a mechanical refining process with a higher energy input caused more severe breakage of wood fiber with shorter length and width [34]. Additionally, Karinkanta et al., demonstrated that smaller particles were obtained at conditions with higher impact energy input when Norway spruce was pulverized with an oscillatory ball mill [29]. We also observe that $d_{50}$ approached around $20 \mu \mathrm{m}$ at 6-minutes milling time under all tested moisture content conditions, while the changes in $d_{50}$ were slight for additional milling time to 12 minutes. These systematic changes in particle size with respect to milling time are in agreement with the literature reports, which claimed that particle size could not infinitely decrease by prolonging milling time after attaining a critical size [22]. The width of particle size distribution $\Delta_{d}$ is increased as a function of $d_{50}$ for samples with different initial moisture contents (Fig. 1B). The strong linear decrease in $\Delta_{d}$ with respect to $d_{50}$ suggests that the mechanical milling resulted in effective particle size reduction of micronized wood, yielding more homogeneous particles.

The median particle size $d_{50}$ of micronized wood produced from various feedstock sizes is depicted in Fig. 1C. For different feed size samples, we successfully comminuted the particles from the millimeter to micrometer range in 12-minutes or less, using a highly versatile and energy-efficient ring and puck milling system. Micronizing woody biomass from a chip size (i.e., P25 in Fig. 1C) can thus be effectively achieved in relatively a short milling time compared to the ball milling times of several hours or days reported by others $[14,35,36]$. At an early stage of the milling process, we notice that feedstock with various initial sizes were milled to similar 
size around $100-\mu \mathrm{m}$. This behavior was likely a result of a higher contact area upon impact for larger-sized samples during milling process. A previous study on impact milling of lignocellulosic biomass indicated that larger particles experienced a higher breakage probability, since the internal failure probability increases with volume as well as with the particle's contact area upon impact [11]. We also observe that a rapid decrease of particles reaching ca. $20 \mu \mathrm{m}$ for various initial sizes at 6-minutes milling time point, followed by a slight change of particle size after further milling to 12 minutes. The similar tendency of particle size evolution from different input sizes suggests that initial size has a negligible effect on development of micronized wood particle size, even though bigger size sample breaks up faster at early stage of milling process. The relationship between the width $\Delta_{d}$ and mean $d_{50}$ of the particle size distribution for samples with different feed sizes is presented in Fig.1D. The results also demonstrate effective size reduction and generation of homogeneous particles where the $\Delta_{d}$ decreases linearly with $d_{50}$ (with high coefficient $\mathrm{R}^{2}=0.98$ ).

\section{Insert Fig. 1 here}

Fig. 1. Effect of milling conditions on the median particle size and width of particle size distribution. (A) median particle size changes of samples with different moisture contents; (B) width of particle size distribution of samples with different moisture contents; (C) median particle size changes of samples with different feed sizes; (D) width of particle size distribution of samples with different feed sizes;

The influence of milling conditions on changes to the shape of micronized particles, as represented by median aspect ratio $A R_{50}$ and width distribution of aspect ratio $\Delta_{\mathrm{AR}}$ are shown in Fig. 2. The initial moisture content differentiated the shape development of micronized wood (Fig. 2A). We observe that $A R_{50}$ decreased gradually with the increase of milling time 
for those samples with initial moisture content of 5\%,10\% and 15\%, whereas less change in aspect ratio for samples with initial moisture content of $30 \%$ is noticed. These observations indicate that lower moisture content contributes to much rounder particles, and higher moisture content results in particles with higher aspect ratio. The linear decrease of aspect ratio distribution width $\Delta_{\mathrm{AR}}$ with respect to mean $A R_{50}$ indicates that micronized particles possessed much more uniform shape after mechanical milling. The median aspect ratio $A R_{50}$ of micronized wood milled from various feedstock sizes is depicted in Fig. 1C and D. We notice that the feed size has negligible influence on $A R_{50}$ to milling time relation for micronized wood produced by this type of milling (Fig. 2C). All the samples evolved to similar particle shape during milling process, regardless of their initial size. In addition, the linear relation between the breadth $\Delta_{A R}$ and mean $A R_{50}$ of the particle aspect ratio distribution also suggests homogeneous particle shape of micronized wood regardless of the feed sizes (Fig. 2D).

\section{Insert Fig. 2 here}

Fig. 2. Effect of milling conditions on the median aspect ratio and the width of particle aspect ratio distribution. (A) median aspect ratio changes of samples with different moisture contents; (B) width of aspect ratio distribution of samples with different moisture contents; (C) median aspect ratio changes of samples with different feed sizes; (D) width of aspect ratio distribution of samples with different feed sizes;

\subsection{Effect of milling conditions on cellulose crystallinity}

Besides observing the morphology changes of micronized wood during the milling process, changes in cellulose crystallinity were also characterized. The crystallinity index (CrI) changes of micronized wood produced from various initial moisture contents are 
delineated in Fig. 3A. Overall, CrI decreases significantly with the increase of milling time for those tested samples, suggesting effective disruption of crystalline structure during ring and puck milling process. This is in agreement with other reports on crystallinity reduction of lignocellulosic biomass subjected to mechanical actions (e.g., impacting, and shearing, etc.) with various milling techniques [3,4]. More precisely, the CrI decreased rapidly to around $10 \%$ for micronized wood produced with relative low moisture contents (e.g., MC5\% and MC10\%), suggesting significant amorphization of crystalline cellulose. Nevertheless, the CrI of micronized samples that were milled with a relatively higher initial moisture content (e.g., MC30\%) only decreased to 40\% even after the longest milling time of 12-minutes, suggesting less changes in crystalline structure during milling process. These phenomena indicate that the initial moisture content significantly influenced the development of cellulose crystallinity. Previous research on milling pretreatment of sugarcane bagasse and wheat straw using dry ball and wet disk milling indicated that dry milling resulted in significant amorphozation of these herbaceous biomass feedstock, whereas wet milling only slightly reduced the crystallinity [37]. Hoeger et al., also reported that mechanical fibrillation of softwood pulping fibers in wet state with stone grinding contributed to only $15-25 \%$ crystallinity reduction with fibrillation time of 6-12hours [33]. The CrI changes of micronized wood produced from various initial sizes are depicted in Fig. 3B. There is gradual decrease of $\mathrm{CrI}$ to around $10 \%$ with respect to milling time for samples milled from different feed sizes. The changes in CrI show similar tendency for different feed size samples, suggesting negligible effect of feed size on crystallinity development of micronized wood. These results are consistent with previously reported observations that 
various woody and herbaceous biomass feedstock with different initial sizes were effectively milled to similar crystallinity using a tandem-ring mill [20].

\section{Insert Fig. 3 here}

Fig. 3. Effect of changes in initial moisture content (A) and feed size (B) on development of crystallinity index (CrI) of micronized wood as a function of milling time.

\subsection{Relations of structural characteristics of micronized wood}

Mechanical milling conditions can generate micronized wood with distinctive particle morphology and crystallinity as discussed above. It is also interesting and essential to investigate the relationships among these structural characteristics of micronized wood for better understanding of sequential particle evolution during milling process. Thus, the changes of median aspect ratio $A R_{50}$ as a function of median size $d_{50}$ for those obtained samples under various milling conditions are shown in Fig. 4. It is observed that $A R_{50}$ decreased slightly with the decline of $d_{50}$ for samples with initial moisture content of MC30\%, whereas those from other samples showed drastic decrease. Such phenomena may suggest that the development of micronized wood is mainly related to fracture modes that are determined by initial moisture contents during milling process. It is interpreted that the fiber fragmentation mode is responsible for the development of micronized wood with high moisture content input (e.g., MC30\%). That means the fiber fracture parallel to the fiber length occurred more frequently than it did perpendicular to the wood grain, with higher initial wood moisture content (i.e., 30\%). This fracture behavior resulted in a decrease in particle size with a negligible change in aspect ratio. As elucidated by previous research on mechanical pulping, refining along the fiber length resulted in fibers peeling rather than fracturing across the fiber length [38,39]. During the milling 
process, the lower moisture content samples irrespective of their sizes might undergo chipping and abrasion, leading to shorter fibers along with particle size reduction, collectively.

On the other hand, the strength of wood fibers perpendicular to the cellulose fibrils is significantly higher when wood is below the fiber saturation point; ca. 30\%. This behavior results from increased hydrogen bonding between cellulose chains that would otherwise be bridged by water molecules [40]. With relatively lower moisture contents, wood fibers possessed higher stiffness. Fracture would thus propagate in the direction perpendicular to the fiber length more frequently at low moisture content, reducing aspect ratio along with particle size. In addition, the increased probability of fracture perpendicular to the fiber length was also coincident with the disruption of crystalline structure, resulting in lower cellulose crystallinity for lower moisture content samples.

The difference of particle aspect ratios for milled wood at various moisture contents is also visually evident. SEM images of of micronized particles produced at various moisture contents and a milling time of 12-minutes are shown in Fig. 5. Fibrous particles were obtained from MC30\% sample, while relatively low moisture content samples resulted in glabular and sperical particles.

\section{Insert Fig. 4 here}

Fig. 4. Relation of median aspect ratio and median particle size of micronized wood from various milling conditions.

\section{Insert Fig. 5 here}

Fig. 5. Morphology difference of micronized wood with different initial moisture contents (A: MC5\%, B: MC10\%, C: MC15\%, D: MC30\%) during milling process; Feed size: P3; Loading: 10 g; Milling time: 12 minutes. 
The relations between cellulose crystallinity and particle morphology are depicted in Fig.

6. The CrI decreases significantly with the decrease of median particle size $d_{50}$ for all milling conditions (Figure 2.6A). Nevertheless, there is less change of CrI for samples milled with relatively higher moisture content (e.g., MC30\%) compared to all others. This decreased change in crystallinity is noted despite the fact that the resulting particles were smaller than those produced at the lower moisture contents. This observation is in agreement with previous reports, which claimed that the moisture content of lignocellulosic substrates was a primary factor affecting the decrease in crystallinity during ball milling [10,41].

Moisture content is an important factor influencing the glass transition of amorphous polymers in wood cell wall matrix, and the glass transition temperatue ( $\mathrm{Tg}$ ) shifts to lower temperature range when moisture content increases $[42,43]$. With the moisture content around $30 \%$, hemicellulose has a $\mathrm{Tg}$ around $-22^{\circ} \mathrm{C}$, thus existing in its rubbery state with greatly reduced stiffness at ambient milling condition. The $\mathrm{Tg}$ of hemicellulose is about $30^{\circ} \mathrm{C}$ at approximately $10 \%$ moisture content [42]. That means the amorphous polymers were in their glass state during ambient milling of low moisture content samples. Wood fiber structure is also known to have microfibril bundles embedded within the amorphous polymers matrix [44]. Thus, during the milling process, the fracture of wood fibers occuring in higher moisture content samples would primarily include the breakage of rubbery state matrix and splitting apart of microfibril bundles without causing extensive disorder within the crystalline structure of cellulose. However, for the lower moisture content samples, the impact milling disrupted the brittle cell wall matrix and crystalline cellulose skeleton. This led to a collective decrease of particle size and cellulose crystallinity, which is in aggreement with the previously discussed 
evolution of particle aspect ratio. Fig. 6B illustrates the strong linear relationship between $A R_{50}$ and CrI, regardless of the moisture content and initial size. The quality of the linear fit is characterized by high coefficients $\left(\mathrm{R}^{2}=0.90\right)$. Here, larger aspect ratio values correspond to higher CrI.

\section{Insert Fig. 6 here}

Fig .6. Development of crystallinity index (CrI) as a function of (A) median particle size and (B) median aspect ratio of micronized wood from various milling conditions.

\subsection{Analysis of energy requirements for size reduction}

Besides the physical properties of micronized particles, specific energy consumption for producing such particles is another important factor for evaluating fine milling performance of woody biomass. Rittinger's model is an appropriate model to describe the fine milling performance, where the new surface area generated per unit mass of the particle is directly proportional to the specific energy consumption for size reduction [25-27]. The specific surface area is inversely proportional to the particle size. Therefore, the Rittinger's model can be

expressed as follows: $E=C\left(\frac{1}{d_{p}}-\frac{1}{d_{f}}\right)$, where $\mathrm{C}$ is a constant characteristic of the material,

$d_{p}$ and $d_{f}$ are the characteristic particle size of milled product and feed [45]. The advantage of applying this model lies in characterizing the grinding performance of woody biomass with one parameter, which can be determined from model constant C. The collection of limited parameters such as specific energy consumption and particle size will benefit to scale-up processing of fine milling of woody biomass. 
Linear relationships between specific energy consumption and particle size reduction parameter in Rittinger's model $\left(1 / d_{p}-1 / d_{f}\right)$ for samples with different initial moisture contents are shown in Fig. 7. The quality of the linear fit is characterized by high coefficients $\left(0.91<\mathrm{R}^{2}<\right.$ 0.95). In previous study, Karinkanta et al. also reported linear relationship between the inverse value of $d_{50}$ of finely ground wood powder and total available impact energy with oscillatory ball mill in accordance to Rittinger's model [29]. The model constant $\mathrm{C}$ can be a good indicator for grindability of woody biomass, and for evaluating the energy efficiency of milling process when similar materials are used as a probe [46]. As discussed above, moisture content was the key factor influencing the development of morphology and crystallinity of micronized wood, and to a large extent, the specific energy consumption. Increasing the moisture content induced increase of the specific energy consumption during milling process, as indicated by model constant values. This phenomenon was in agreement with previous research on energy consumption for milling various wood species with different moisture contents conducted using laboratory-scale hammer mill [46]. As indicated above, moisture content greatly affects the toughness of woody biomass. At low initial moisture content (e.g., MC 5\%), the brittle polymer components fractured easily with low energy consumption. Increasing the moisture content resulted in the amorphous polymers becoming tougher due to the moisture acting as a plasticizer, thus requiring more energy consumption on fatigue to cause rupturing.

\section{Insert Fig. 7 here}

Fig. 7. Effect of moisture content on specific net energy consumption as a function of particle size reduction $\left(1 / d_{p}\right.$ $\left.1 / d_{f}\right)$ 
The specific energy consumption as a function of size reduction parameter $\left(1 / d_{p}-1 / d_{f}\right)$ in Rittinger's model for various initial size samples is shown in Fig. 8. The experimental results are also fitted well to Rittinger's model. The linear relations are identified with high coefficients $\left(0.91<\mathrm{R}^{2}<0.96\right)$. The larger the feed size, the more energy was required for fine milling. Although ring and puck mill is capable of comminuting chip-sized feedstock to micrometer range within minutes, it is not economically viable from the energy perspective. Coarse milling of Douglas-fir with different fitted screen sizes as used in the current study through pilot-scale hammer mill had been carried out in our lab, and the results indicated that the energy consumption for milling wood chips was in the range of $59-141 \mathrm{~kJ} / \mathrm{kg}$ depending on specific fitted screen size. Several researchers also reported that fine milling of woody biomass was much more energy consuming than coarse milling $[4,21,46,47]$. Therefore, multi-step comminution strategy with combination of coarse and fine milling seems much more economical than directly fine milling of large-sized feedstock. However, the multi-step comminution will inevitably increase the capital investments.

The difference in energy consumption for milling samples with various properties suggests that minimizing the grindability of woody biomass is necessary when using ring and puck mill for fine milling. Therefore, it is reasonable to standardize the milling variables using a reference standard (e.g. chemical composition, moisture content and size) to obtain the grindability of lab-scale test as reported in mineral and biomass industry [48-50]. In addition, our results show very competitive potential for industrial scale production of fine wood powders with such comminution technology. Disk milling predominately used in paper pulping area for wood fiber disintegration (i.e., size reduction) generally requires energy consumption of over 2 $\mathrm{MJ} / \mathrm{kg}$ wood depending on wood species and the size reduction ratios [51]. Kobayashi et al. [10] 
have reported that the energy requirement for pulverizing $22 \mathrm{~mm}$-size Norway spruce wood chips into powders with a size of $150 \mu \mathrm{m}$ is $2.95 \mathrm{MJ} / \mathrm{kg}$ (including conveyer, compressor and powder sieve) using a continuous vibration mill. When dry spruce sawdust with size range around $1 \mathrm{~mm}$ was comminuted with an oscillatory ball mill, Karinkanta et al. [29] found that the energy consumption of $0.36 \mathrm{MJ} / \mathrm{kg}$ was needed to get wood particles with size around $100 \mu \mathrm{m}$ and 1.4 $\mathrm{MJ} / \mathrm{kg}$ was consumed to get wood powder with size around $20 \mu \mathrm{m}$. In this study, pulverization of Douglas-fir chips with moisture content of 5\% and initial size about $1 \mathrm{~mm}$ (i.e., feed sample P3) down to size around $100 \mu \mathrm{m}$ is accomplished with energy consumption of $0.28 \mathrm{MJ} / \mathrm{kg}$, while size reduction to $20 \mu \mathrm{m}$ is accomplished with energy consumption of $0.86 \mathrm{MJ} / \mathrm{kg}$. Therefore, the energy consumption for wood particles pulverization provides a practical target for optimization of comminuting woody biomass into micron size range for industrial application using ring and puck mill.

\section{Insert Fig. 8 here}

Fig. 8. Effect of feed size on specific net energy consumption as a function of particle size reduction $\left(1 / d_{p}-1 / d_{f}\right)$.

\section{Conclusions}

We effectively micronized softwood Douglas-fir using a vibratory ring and puck mill within several minutes. The effect of substrate properties (i.e., moisture content and initial size) on development of physical properties of micronized particles and specific energy consumption for producing such particles were investigated. Results indicated that moisture content was the most important factor influencing milling behavior in terms of particle morphology and cellulose crystallinity. The lower moisture content samples resulted in much rounder particles with 
relatively smaller crystallinity than their higher moisture counterparts. The feed size had negligible influence on development of particle physical properties, but only affected the specific energy consumption. A multi-step milling strategy is recommended for a more economical approach for fine milling of woody biomass. The crystallinity and aspect ratio of particles underwent similar development tendency. There was a linear relationship between crystallinity index and median aspect ratio of milled particles.

Rittinger's model was ideal for describing the relationship between specific energy consumption, and particle size changes. The constant in this model was a good indicator of grindability of woody biomass with different properties. It is also recommended that standardizing milling test and parameters could benefit to evaluating the grinding performance of fibrous materials on a lab-scale, as such information would be useful for designing scale-up milling facilities of woody and herbaceous biomass feedstock.

\section{List of symbols}

$d_{50} \quad$ Median particle size $(\mu \mathrm{m})$

$\Delta_{d} \quad$ The breadth of the particle size distribution (unitless)

$A R_{50}$ The value of $50 \%$ cumulative aspect ratio distribution (unitless)

$\Delta_{A R}$ The difference between the aspect ratios corresponding to $90 \%$ and $10 \%$ values in the cumulative distribution

$E_{p} \quad$ The specific net energy consumption $(\mathrm{kJ} / \mathrm{kg}$ or $\mathrm{MJ} / \mathrm{kg}$ )

$\mathrm{t} \quad$ The milling time $(\mathrm{s})$

$P_{t} \quad$ The power consumed at time $\mathrm{t}(\mathrm{W})$

$P_{0} \quad$ The average power consumption under idle condition measured from an empty mill (W)

$\mathrm{m} \quad$ The mass charge in $\mathrm{kg}$ of wood $(\mathrm{kg})$

\section{Acknowledgements}

The authors are grateful to the financial support from the Agriculture and Food Research Initiative (AFRI) Competitive grant (No. 2011-68005-30416), USDA National Institute of Food and Agriculture (NIFA) through the Northwest Advanced Renewables Alliance (NARA). The 
authors would also like to acknowledge the help of scanning electronic microscopy analysis from

Franceschi Microscopy \& Imaging Center (FMIC) at Washington State University, Pullman.

\section{Reference}

[1] P. Alvira, E. Tomás-Pejó, M. Ballesteros, M.J. Negro, Pretreatment technologies for an efficient bioethanol production process based on enzymatic hydrolysis: A review, Bioresour. Technol. 101 (2010) 4851-4861. doi:10.1016/j.biortech.2009.11.093.

[2] P.L. Eranki, B.D. Bals, B.E. Dale, Advanced Regional Biomass Processing Depots: a key to the logistical challenges of the cellulosic biofuel industry, Biofuels Bioprod. Biorefining. 5 (2011) 621-630.

[3] A. Barakat, C. Mayer-Laigle, A. Solhy, R.A.D. Arancon, H. de Vries, R. Luque, Mechanical pretreatments of lignocellulosic biomass: towards facile and environmentally sound technologies for biofuels production, RSC Adv. 4 (2014) 48109-48127. doi:10.1039/C4RA07568D.

[4] A. Barakat, H. de Vries, X. Rouau, Dry fractionation process as an important step in current and future lignocellulose biorefineries: A review, Bioresour. Technol. 134 (2013) 362-373. doi:10.1016/j.biortech.2013.01.169.

[5] P. Langan, L. Petridis, H.M. O’Neill, S.V. Pingali, M. Foston, Y. Nishiyama, R. Schulz, B. Lindner, B.L. Hanson, S. Harton, W.T. Heller, V. Urban, B.R. Evans, S. Gnanakaran, A.J. Ragauskas, J.C. Smith, B.H. Davison, Common processes drive the thermochemical pretreatment of lignocellulosic biomass, Green Chem. 16 (2014) 63. doi:10.1039/c3gc41962b.

[6] H. Lu, E. Ip, J. Scott, P. Foster, M. Vickers, L.L. Baxter, Effects of particle shape and size on devolatilization of biomass particle, Fuel. 89 (2010) 1156-1168. doi:10.1016/j.fuel.2008.10.023.

[7] Z. Miao, T.E. Grift, A.C. Hansen, K.C. Ting, Energy requirement for comminution of biomass in relation to particle physical properties, Ind. Crops Prod. 33 (2011) 504-513. doi:10.1016/j.indcrop.2010.12.016.

[8] A.A. Modenbach, S.E. Nokes, The use of high-solids loadings in biomass pretreatment-a review, Biotechnol. Bioeng. 109 (2012) 1430-1442. doi:10.1002/bit.24464.

[9] J. Jiang, J. Wang, X. Zhang, M. Wolcott, Evaluation of physical structural features on influencing enzymatic hydrolysis efficiency of micronized wood, RSC Adv. 6 (2016) 103026-103034. doi:10.1039/C6RA22371K.

[10] N. Kobayashi, T. Sato, N. Okada, J. Kobayashi, S. Hatano, Y. Itaya, S. Mori, Evaluation of wood powder property pulverized by a vibration mill, J. Jpn. Institue Energy. 86 (2007) 730-735.

[11] M. Gil, E. Luciano, I. Arauzo, Approach to the breakage behavior of comminuted poplar and corn stover under single impact, Fuel Process. Technol. 131 (2015) 142-149. doi:10.1016/j.fuproc.2014.11.020.

[12] V.S.P. Bitra, A.R. Womac, N. Chevanan, P.I. Miu, C. Igathinathane, S. Sokhansanj, D.R. Smith, Direct mechanical energy measures of hammer mill comminution of switchgrass, 
wheat straw, and corn stover and analysis of their particle size distributions, Powder Technol. 193 (2009) 32-45. doi:10.1016/j.powtec.2009.02.010.

[13] G.G.D. Silva, M. Couturier, J.-G. Berrin, A. Buléon, X. Rouau, Effects of grinding processes on enzymatic degradation of wheat straw, Bioresour. Technol. 103 (2012) 192200. doi:10.1016/j.biortech.2011.09.073.

[14] M.R. Zakaria, S. Fujimoto, S. Hirata, M.A. Hassan, Ball Milling Pretreatment of Oil Palm Biomass for Enhancing Enzymatic Hydrolysis, Appl. Biochem. Biotechnol. (2014). doi:10.1007/s12010-014-0964-5.

[15] J. Jiang, J. Wang, X. Zhang, M. Wolcott, Microstructure change in wood cell wall fracture from mechanical pretreatment and its influence on enzymatic hydrolysis, Ind. Crops Prod. 97 (2017) 498-508. doi:10.1016/j.indcrop.2017.01.001.

[16] Z. Wang, A.G. McDonald, R.J.M. Westerhof, S.R.A. Kersten, C.M. Cuba-Torres, S. Ha, B. Pecha, M. Garcia-Perez, Effect of cellulose crystallinity on the formation of a liquid intermediate and on product distribution during pyrolysis, J. Anal. Appl. Pyrolysis. 100 (2013) 56-66. doi:10.1016/j.jaap.2012.11.017.

[17] P. Smith, M. Wolcott, Opportunities for wood/natural fiber-plastic composites in residential and industrial applications, For. Prod. J. 56 (2006) 4-11.

[18] J. Kobayashi, Y. Itaya, S. Tsukada, K. Mizuno, M. Ueda, H. Morikawa, T. Sugimoto, Y. Ueda, Y. Oshika, N. Kobayashi, others, Drying technology for woody biomass for fine grinding by vibration mills, Asia-Pac. J. Chem. Eng. 2 (2007) 83-89.

[19] H. Peng, H. Li, H. Luo, J. Xu, A novel combined pretreatment of ball milling and microwave irradiation for enhancing enzymatic hydrolysis of microcrystalline cellulose, Bioresour. Technol. 130 (2013) 81-87. doi:10.1016/j.biortech.2012.10.167.

[20] T. Takahashi, K. Ito, A. Ito, Y. Enda, M. Gochi, H. Mori, J. Kobayashi, Tandem-ring mill pulverization benefits for enzymatic saccharification of biomass, Renew. Energy. (2013). doi:10.1016/j.renene.2013.07.046.

[21] V. Repellin, A. Govin, M. Rolland, R. Guyonnet, Energy requirement for fine grinding of torrefied wood, Biomass Bioenergy. 34 (2010) 923-930. doi:10.1016/j.biombioe.2010.01.039.

[22] P. Karinkanta, M. Illikainen, J. Niinimäki, Effect of different impact events in fine grinding mills on the development of the physical properties of dried Norway spruce (Picea abies) wood in pulverisation, Powder Technol. 253 (2014) 352-359. doi:10.1016/j.powtec.2013.11.044.

[23] S. Mani, L.G. Tabil, S. Sokhansanj, Grinding performance and physical properties of wheat and barley straws, corn stover and switchgrass, Biomass Bioenergy. 27 (2004) 339-352. doi:10.1016/j.biombioe.2004.03.007.

[24] Z. Miao, Y. Shastri, T.E. Grift, A.C. Hansen, K.C. Ting, Lignocellulosic biomass feedstock transportation alternatives, logistics, equipment configurations, and modeling, Biofuels Bioprod. Biorefining. 6 (2012) 351-362.

[25] A. Wisconsin, Factors contributing to the strength properties of a sheet of paper, 1957.

[26] P.J. Fellows, Food Processing Technology - Principles and Practice, 3rd ed., Woodhead Publishing, Cambridge, 2000.

[27] Y. Chen, Y. Ding, D.G. Papadopoulos, M. Ghadiri, Energy-based analysis of milling $\alpha-$ lactose monohydrate, J. Pharm. Sci. 93 (2004) 886-895.

[28] A. Sluiter, B. Hames, D. Hyman, C. Payne, R. Ruiz, C. Scarlata, J. Sluiter, D. Templeton, J. Wolfe, Determination of total solids in biomass and total dissolved solids in liquid process 
samples, Natl. Renew. Energy Lab. Colo. (2008).

http://www.nrel.gov/docs/gen/fy08/42621.pdf (accessed May 31, 2013).

[29] P. Karinkanta, M. Illikainen, J. Niinimäki, Impact-based pulverisation of dried and screened Norway spruce (Picea abies) sawdust in an oscillatory ball mill, Powder Technol. 233 (2013) 286-294. doi:10.1016/j.powtec.2012.09.011.

[30] C. Igathinathane, S. Melin, S. Sokhansanj, X. Bi, C.J. Lim, L.O. Pordesimo, E.P. Columbus, Machine vision based particle size and size distribution determination of airborne dust particles of wood and bark pellets, Powder Technol. 196 (2009) 202-212. doi:10.1016/j.powtec.2009.07.024.

[31] A. Mazzoli, O. Favoni, Particle size, size distribution and morphological evaluation of airborne dust particles of diverse woods by Scanning Electron Microscopy and image processing program, Powder Technol. 225 (2012) 65-71. doi:10.1016/j.powtec.2012.03.033.

[32] L. Segal, J.J. Creely, A.E. Martin, C.M. Conrad, An Empirical Method for Estimating the Degree of Crystallinity of Native Cellulose Using the X-Ray Diffractometer, Text. Res. J. 29 (1959) 786-794. doi:10.1177/004051755902901003.

[33] I.C. Hoeger, S.S. Nair, A.J. Ragauskas, Y. Deng, O.J. Rojas, J.Y. Zhu, Mechanical deconstruction of lignocellulose cell walls and their enzymatic saccharification, Cellulose. 20 (2013) 807-818. doi:10.1007/s10570-013-9867-9.

[34] D. Fernando, D. Muhić, P. Engstrand, G. Daniel, Fundamental understanding of pulp property development under different thermomechanical pulp refining conditions as observed by a new Simons' staining method and SEM observation of the ultrastructure of fibre surfaces, Holzforschung. 65 (2011) 777-786. doi:10.1515/HF.2011.076.

[35] Z. Hu, Elucidation of the Structure of Cellulolytic Enzyme Lignin from Loblolly Pine (pinus taeda), Master of Science, North Carolina State University, 2006. http://repository.lib.ncsu.edu/ir/handle/1840.16/26 (accessed May 20, 2014).

[36] H.J. Kim, S. Lee, J. Kim, R.J. Mitchell, J.H. Lee, Environmentally friendly pretreatment of plant biomass by planetary and attrition milling, Bioresour. Technol. 144 (2013) 50-56. doi:10.1016/j.biortech.2013.06.090.

[37] A.S. da Silva, H. Inoue, T. Endo, S. Yano, E.P.S. Bon, Milling pretreatment of sugarcane bagasse and straw for enzymatic hydrolysis and ethanol fermentation, Bioresour. Technol. 101 (2010) 7402-7409. doi:10.1016/j.biortech.2010.05.008.

[38] A. Karnis, The mechanism of fibre development in mechanical pulping, J Pulp Pap. Sci. 20 (1994) 280-288.

[39] W.Y. Hamad, Some microrheological aspects of wood-pulp fibres subjected to fatigue loading, Cellulose. 4 (1997) 51-56.

[40] M.F. Ashby, K.E. Easterling, R. Harrysson, S.K. Maiti, The Fracture and Toughness of Woods, Proc. R. Soc. Lond. Ser. Math. Phys. Sci. 398 (1985) 261-280.

[41] P. Karinkanta, M. Illikainen, J. Niinimäki, Effect of grinding conditions in oscillatory ball milling on the morphology of particles and cellulose crystallinity of Norway spruce (Picea abies), Holzforschung. 67 (2013) 277-283. doi:10.1515/hf-2012-0098.

[42] S.S. Kelley, T.G. Rials, W.G. Glasser, Relaxation behaviour of the amorphous components of wood, J. Mater. Sci. 22 (1987) 617-624.

[43] M.P. Wolcott, F.A. Kamke, D.A. Dillard, Fundamental aspects of wood deformation pertaining to manufacture of wood-based composites, Wood Fiber Sci. 26 (1994) 496-511. 
[44] B. Yang, Z. Dai, S.-Y. Ding, C.E. Wyman, Enzymatic hydrolysis of cellulosic biomass, Biofuels. 2 (2011) 421-450. doi:10.4155/bfs.11.116.

[45] A. Jankovic, H. Dundar, R. Mehta, Relationships between comminution energy and product size for a magnetite ore, J. South Afr. Inst. Min. Metall. 110 (2010) 141.

[46] M. Temmerman, P.D. Jensen, J. Hébert, Von Rittinger theory adapted to wood chip and pellet milling, in a laboratory scale hammermill, Biomass Bioenergy. 56 (2013) 70-81. doi:10.1016/j.biombioe.2013.04.020.

[47] M. Phanphanich, S. Mani, Impact of torrefaction on the grindability and fuel characteristics of forest biomass, Bioresour. Technol. 102 (2011) 1246-1253. doi:10.1016/j.biortech.2010.08.028.

[48] M.M. Ahmed, Effect of comminution on particle shape and surface roughness and their relation to flotation process, Int. J. Miner. Process. 94 (2010) 180-191. doi:10.1016/j.minpro.2010.02.007.

[49] E. Doroodchi, H. Zulfiqar, B. Moghtaderi, A combined experimental and theoretical study on laboratory-scale comminution of coal and biomass blends, Powder Technol. 235 (2013) 412-421. doi:10.1016/j.powtec.2012.10.054.

[50] D.T. Van Essendelft, X. Zhou, B.S.-J. Kang, Grindability determination of torrefied biomass materials using the Hybrid Work Index, Fuel. 105 (2013) 103-111. doi:10.1016/j.fuel.2012.06.008.

[51] J.Y. Zhu, Physical Pretreatment-Woody Biomass Size Reduction-Forest Biorefinery, in: Sustain. Prod. Fuels Chem. Fibers For. Biomass, American Chemical Society, 2011. 